\title{
High Frequency Pulse Propagation in Nonlinear Dielectric Materials
}

\author{
H.T. Banks \\ Center for Research in Scientific Computation \\ North Carolina State University \\ Raleigh, NC 27695-8205 \\ Gabriella A. Pinter \\ Department of Mathematical Sciences \\ University of Wisconsin, Milwaukee \\ Milwaukee, WI 53201-0413
}

November 21, 2003

\begin{abstract}
We consider a variational formulation based on Maxwell's equations for the propagation of high frequency (gigahertz to terahertz) ultrashort input pulses in dielectric materials modeled by a linear Debye medium. We demonstrate computationally the emergence of Brillouin precursors in the material (water) and the fact that the peak of this transient is attenuated at a much slower rate than is the carrier frequency. In the 0.1 to $1 \mathrm{THz}$ regime the carrier frequency does not propagate in our calculations. Only the precursors enter the material, and this is in line with experiments reported by Pleshko and Palocz [11]. We also implement models that include nonlinearly forced Debye and nonlinear Debye polarization dynamics and demonstrate the importance of nonlinear effects, especially when the amplitude of the input signal is large. This is an important step in understanding high frequency pulse propagation, and it has potential applications in the assessment of safety standards and in extending current imaging capabilities in both civilian and military uses.
\end{abstract}

\section{Introduction}

In recent years there has been a great technological development in methodology for generating ultrashort pulses of light in the terahertz range. A single pulse of a few picoseconds long allows the full terahertz spectrum to be measured, and thus facilitates better (spatial and electromagnetic) and faster imaging results than more traditional 
techniques. In this frequency range several organic materials exhibit strong absorption and dispersion that are characteristic to the particular molecular structure and content. Thus, detection of the temporal distortions of the electromagnetic wave caused by the interaction yields information about the composition of the material in real time. For example, it is known that cancerous and benign tumors have different electromagnetic characteristics. Thus an imaging device based on $\mathrm{THz}$ waves could not only give information about the structure of an object (geometrical properties) but could help in determining its composition and electromagnetic properties as well in a non-invasive way.

In this paper we present extensive numerical simulations modeling the propagation of electromagnetic waves in the gigahertz to terahertz frequency range through dielectric materials with special emphasis on ultrashort input pulses. In the first set of numerical experiments we concentrate on a linear Debye medium, and study the propagation characteristics, in particular, temporal transients such as the Brillouin precursors as they change with the increasing carrier frequency of the input pulse. Our results are in agreement with theoretical and experimental observations [8, 11]. It is known that most materials exhibit nonlinear characteristics in their interaction with the electromagnetic wave, especially when the input signal has a large amplitude [2]. Our models incorporate different nonlinear polarization mechanisms which influence the propagation characteristics. In particular, we study a nonlinearly forced linear Debye model [6], as well as a mechanistically nonlinear Debye model. Our computational framework is based on a variational formulation of Maxwell's equations. We believe that this framework provides a means to capture important dynamic phenomena associated with short input pulses (which has been demonstrated for microwave propagation), and is amenable to both theoretical and computational investigations. The simulations reported here form an important step towards using electromagnetic pulses for interrogation of unknown materials with potential applications in industrial inspection, security screening, medical diagnostics and several other civilian and military problems.

The paper is organized as follows: in Section 2 we present the general variational framework describing the physical problem, we outline the numerical methods used and present the results for a linear Debye medium. In Section 3 we include nonlinearities in the form of a nonlinearly forced Debye polarization mechanism, while in Section 4 we present our initial results for a nonlinear Debye model.

\section{Variational framework and linear results}

In this section we describe a model that was initially developed for the propagation of microwaves in dielectric materials in the monograph [3]. The authors studied both the direct and the inverse problem, and investigated the feasibility of using windowed pulses from antenna sources to interrogate the material. They developed a theoretical and computational framework for using reflected signals to identify both geometric and electromagnetic properties of an object. The inverse problems were solved either by use of a supraconductive backing behind the object or by use of acoustic waves from which the signal is reflected. 
Our focus here is on understanding the propagation characteristics of pulses with different carrier frequencies, and for this purpose we set up a simplified model. However, we note that this simplified formulation can be readily extended to treat interfaces and the more general inverse problems. We consider as in [3] an infinite (in the $x$ and $y$ direction) slab of homogeneous material of width $\ell$ with faces parallel to the $x y$ plane. However, here we place an antenna inside the material, usually in the middle. The input signal is a planar electromagnetic wave polarized with oscillations in the $x z$ plane only. The electromagnetic field $\vec{E}$ is reduced to one nontrivial component, in the $x$ direction, at all points of the slab, and it is homogeneous in intensity in the $x$ and $y$ directions. The electromagnetic flux density $\vec{D}$ and the polarization $\vec{P}$ inherit this uniform directional property from $\vec{E}$. With these assumptions Maxwell's equations yield

$$
\mu_{0} \varepsilon_{0} \epsilon_{r} \ddot{E}+\mu_{0} \ddot{P}+\mu_{0} \sigma \dot{E}-E^{\prime \prime}=-\mu_{0} \dot{J}_{s} \quad t>0,0<z<\ell,
$$

where $E=E(t, z)$ and $P=P(t, z)$ denote the $x$ components of the electric field and the polarization, respectively, and $D=\varepsilon_{0} \varepsilon_{r} E+P$. The parameter $\mu_{0}$ is the magnetic permeability, $\varepsilon_{0}$ is the electric permittivity of free space and $\varepsilon_{r}$ is the relative permittivity (see [3] for discussions). Here $J_{s}$ is the source current density, while the conduction current density, $J_{c}$, is assumed to be given by Ohm's law as $J_{c}=\sigma E$. It is argued in $[1,3]$ that this simple form of Ohm's law together with the introduction of nonlocality in time through an electric susceptibility kernel (polarization dynamics) capture the frequency dependence of the conductivity of dispersive media. In this section we consider a linear polarization mechanism proposed by Debye [7] to model the behavior of materials with a permanent dipole moment, e.g., water (orientational polarization). This is given by

$$
\tau \dot{P}+P=\varepsilon_{0}\left(\varepsilon_{s}-\varepsilon_{\infty}\right) E, \quad t>0,
$$

where $\varepsilon_{s}$ is the static relative permittivity, while $\varepsilon_{\infty}=\varepsilon_{r}$. We note that this model can be realized with electric susceptibility kernel $g(t)=e^{-t / \tau} \varepsilon_{0}\left(\varepsilon_{s}-\varepsilon_{\infty}\right) / \tau$ in the convolution expression $P=g * E$. For our numerical computations we use approximately absorbing boundary conditions at the ends of the slab to prevent large reflections of the waves

$$
\begin{aligned}
& {\left[\frac{\sqrt{\varepsilon_{s}}}{c} \dot{E}-E^{\prime}\right]_{z=0}=0,} \\
& {\left[\frac{\sqrt{\varepsilon_{s}}}{c} \dot{E}+E^{\prime}\right]_{z=\ell}=0,}
\end{aligned}
$$

with $c^{2} \equiv \frac{1}{\varepsilon_{0} \mu_{0}}$. The approximate nature of these boundary conditions does not cause a problem for us, since in each case we make sure that the computational domain is sufficiently large, so that we do not see any reflections in our time frame. We complete the system with initial conditions

$$
\begin{aligned}
& E(0, z)=0, \quad 0<z<\ell, \\
& \dot{E}(0, z)=0, \quad 0<z<\ell, \\
& P(0, z)=0, \quad 0<z<\ell .
\end{aligned}
$$


We define $V=H^{1}(0, \ell), H=L^{2}(0, \ell)$ and write (2.1) and (2.2) in weak form as

$$
\begin{aligned}
& \left\langle\mu_{0} \varepsilon_{0} \epsilon_{r} \ddot{E}, \varphi\right\rangle+\left\langle\mu_{0} \sigma \dot{E}, \varphi\right\rangle+\langle\ddot{P}, \varphi\rangle+\left\langle E^{\prime}, \varphi^{\prime}\right\rangle+\frac{\sqrt{\varepsilon_{s}}}{c} \dot{E}(0) \varphi(0)+\frac{\sqrt{\varepsilon_{s}}}{c} \dot{E}(\ell) \varphi(\ell) \\
& =-\mu_{0}\left\langle\dot{J}_{s}, \varphi\right\rangle \\
& \langle\tau \dot{P}+P, \varphi\rangle=\left\langle\varepsilon_{0}\left(\varepsilon_{s}-\varepsilon_{\infty}\right) E, \varphi\right\rangle
\end{aligned}
$$

for all $\varphi \in V$. Well-posedness results given in Chapter 3 of [3] can be used to guarantee the usual existence, uniqueness and continuous dependence of solutions for this system.

For computational purposes we scale the time variable by a factor of $c$ and polarization $P$ by a factor of $1 / \varepsilon_{0}$, i.e., we let $\tilde{E}=E(c t), \tilde{P}=1 / \varepsilon_{0} P(c t)$. We express $\ddot{P}$ from (2.2) and substitute it into (2.8). The new equations in the scaled variables are (where for the sake of simplicity of notation we drop the overtildes)

$$
\begin{aligned}
& \left\langle\epsilon_{r} \ddot{E}, \varphi\right\rangle+\left\langle\left(\eta \sigma+\varepsilon_{d} \lambda\right) \dot{E}, \varphi\right\rangle+\left\langle\lambda^{2} P, \varphi\right\rangle-\left\langle\varepsilon_{d} \lambda^{2} E, \varphi\right\rangle+\left\langle E^{\prime}, \varphi^{\prime}\right\rangle+\sqrt{\varepsilon_{s}} \dot{E}(0) \varphi(0) \\
& +\sqrt{\varepsilon_{s}} \dot{E}(\ell) \varphi(\ell)=-\eta\left\langle\dot{J}_{s}, \varphi\right\rangle \\
& \langle\dot{P}+\lambda P, \varphi\rangle=\left\langle\varepsilon_{d} \lambda E, \varphi\right\rangle,
\end{aligned}
$$

where we introduced the notation $\varepsilon_{d}=\varepsilon_{s}-\varepsilon_{\infty}, \lambda=\frac{1}{c \tau}$ and $\eta=\mu_{0} c$. We discretize the problem in the space variable using a first order Galerkin finite element approximation. We divide the interval $[0, \ell]$ into $\mathrm{N}$ equal subintervals at the points $z=j h, j=0, \ldots N$, where $h=\ell / N$, and construct standard piecewise linear splines $\phi_{j}^{N}(z)$. The finite dimensional approximating subspaces to $V$ will be taken to be $V^{N}=\left\{\phi_{0}^{N}, \phi_{1}^{N}, \ldots, \phi_{N}^{N}\right\}$. Now we approximate $E(t, z)$ and $P(t, z)$ in this space as

$$
\begin{aligned}
& E(t, z) \approx E^{N}(t, z)=\sum_{j=0}^{N} e_{j}^{N}(t) \phi_{j}^{N}(z), \\
& P(t, z) \approx P^{N}(t, z)=\sum_{j=0}^{N} p_{j}^{N}(t) \phi_{j}^{N}(z) .
\end{aligned}
$$

We choose the space of test functions to be $V^{N}$ also, and thus we find that (2.10)-(2.11) lead to

$$
\begin{aligned}
& \varepsilon_{r} M \ddot{e}+\left(\left(\eta \sigma+\varepsilon_{d} \lambda\right) M+\sqrt{\varepsilon_{s}} B D\right) \dot{e}+\lambda^{2} M p+\left(K-\lambda^{2} \varepsilon_{d} M\right) e=-\eta \mathcal{J} \\
& M \dot{p}=-\lambda M p+\varepsilon_{d} \lambda M e
\end{aligned}
$$

where $e=\left(e_{0}^{N}, e_{1}^{N}, \ldots, e_{N}^{N}\right)^{T}, \quad p=\left(p_{0}^{N}, p_{1}^{N}, \ldots, p_{N}^{N}\right)^{T}, \quad \mathcal{J}=\left(\mathcal{J}_{0}, \ldots, \mathcal{J}_{N}\right)^{T}$, with $\mathcal{J}_{i}=\left\langle\dot{J}_{s}, \phi_{i}\right\rangle, \quad i=0, \ldots N$, and

$$
\begin{aligned}
M_{i j} & =\left\langle\phi_{i}, \phi_{j}\right\rangle=\int_{0}^{\ell} \phi_{i}(z) \phi_{j}(z) d z, \\
K_{i j} & =\left\langle\phi_{i}^{\prime} \phi_{j}^{\prime}\right\rangle=\int_{0}^{\ell} \phi_{i}^{\prime}(z) \phi_{j}^{\prime}(z) d z .
\end{aligned}
$$


Here $B D$ denotes a matrix with $B D_{00}=B D_{N N}=1$, while all other elements of the matrix are 0 . We now employ a second order central difference approximation in the time variable to solve (2.14). We take

$$
\ddot{e}\left(t^{n}\right)=\ddot{e}^{n} \approx \frac{e^{n+1}-2 e^{n}+e^{n-1}}{\Delta t^{2}}, \dot{e}\left(t^{n}\right)=\dot{e}^{n} \approx \frac{e^{n+1}-e^{n-1}}{2 \Delta t}
$$

and

$$
e\left(t^{n}\right)=e^{n} \approx \frac{e^{n+1}+e^{n}+e^{n-1}}{4} .
$$

Now the Galerkin approximation (2.14) reduces to a linear equation that can be solved for $e^{n+1}$ given $e^{n}, e^{n-1}$ and $p^{n}$. We have initial condition $e^{0}=0$ and we approximate $e^{1} \approx-\eta \frac{\Delta t^{2}}{2 \varepsilon_{r}} \dot{J}_{s}(0, z)$. To obtain $p^{n}$ we solve (2.15) using a Crank-Nicholson method. This approximation method is overall $\mathcal{O}\left(h^{2}\right)$ when $\Delta t=\mathcal{O}(h)$. We also note that the method is unconditionally stable.

In the following we describe our simulations for the problem above. Our physical constants are $\varepsilon_{0}=8.854 \cdot 10^{-12}, c=2.9980 \cdot 10^{8} \mathrm{~m} / \mathrm{sec}, \mu_{0}=1.2566 \cdot 10^{-6}$ We carried out all the calculations assuming that the dielectric material is water with parameters $\varepsilon_{r}=5.5, \varepsilon_{s}=80.1, \tau=8.1 \cdot 10^{-12} \mathrm{sec}, \sigma=10^{-5} \mathrm{Ohm}^{-1}$. We conducted a series of numerical experiments with windowed input signal (generated by an antenna at $z=\ell / 2)$

$$
J_{s}=A \sin ^{3}(\omega t) \chi_{\left[0, t_{f}\right]}(t) \delta(z-\ell / 2),
$$

with amplitude $A=1$, frequencies $\omega=1 \mathrm{GHz}, 10 \mathrm{GHz}, 100 \mathrm{GHz}$ and $1 \mathrm{THz}$, and final input time $t_{f}=10 / \omega$, i.e., we used 5 complete periods of the input signal. We represent the results two different ways: (a) we took snapshots of the electric field in the material at several points in time and (b) we recorded the electric field at particular points in the material from time $t=0$ to some $t=t e n d$. The value of $t e n d$ is chosen in each case such that the input signal reaches the sides of the material, but reflections do not propagate backwards significantly. Figures 1 and 2 depict these simulations for the input signal with carrier frequency $1 \mathrm{GHz}$, while Figures 3 and 4 present the results for the signal with input frequency $10 \mathrm{GHz}$.

We can clearly see the emergence of the Brillouin precursors in both cases. It is important to observe that while the signal is attenuated as it propagates through the material, the rate of attenuation of the transient (precursor) is slower than that of the carrier frequency (which decays exponentially). This fact has important implications for the determination of safety standards since the precursor may penetrate much deeper with more energy than does the carrier frequency part of the signal. The signal attenuation curve is shown in Figure 5 for the $1 \mathrm{GHz}$ case. We remark that these results are in agreement with those reported in [1], where the authors find the electric field in terms of a Fourier series after developing the input pulse train in a series. The drawback of that method is that it does not readily extend to higher frequencies and to the treatment of the inverse or imaging problem. In particular, it is very difficult to treat the interaction of transmitted and reflected waves.

We next report on the results with input frequencies $100 \mathrm{GHz}$ (Figures 6 and 7) and $1 \mathrm{THz}$ (Figures 8 and 9). 

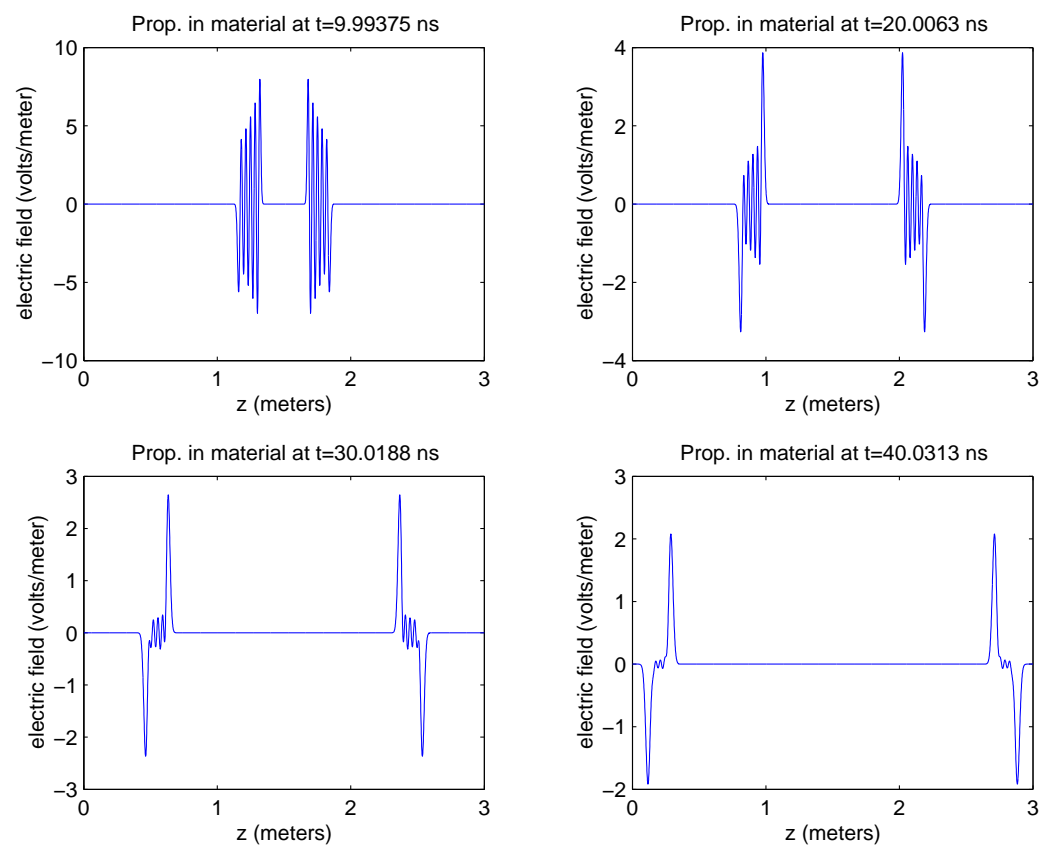

Figure 1: The electric field in the material at $\mathrm{t}=10,20,30$ and $40 \mathrm{~ns}, 1 \mathrm{GHz}$ example.
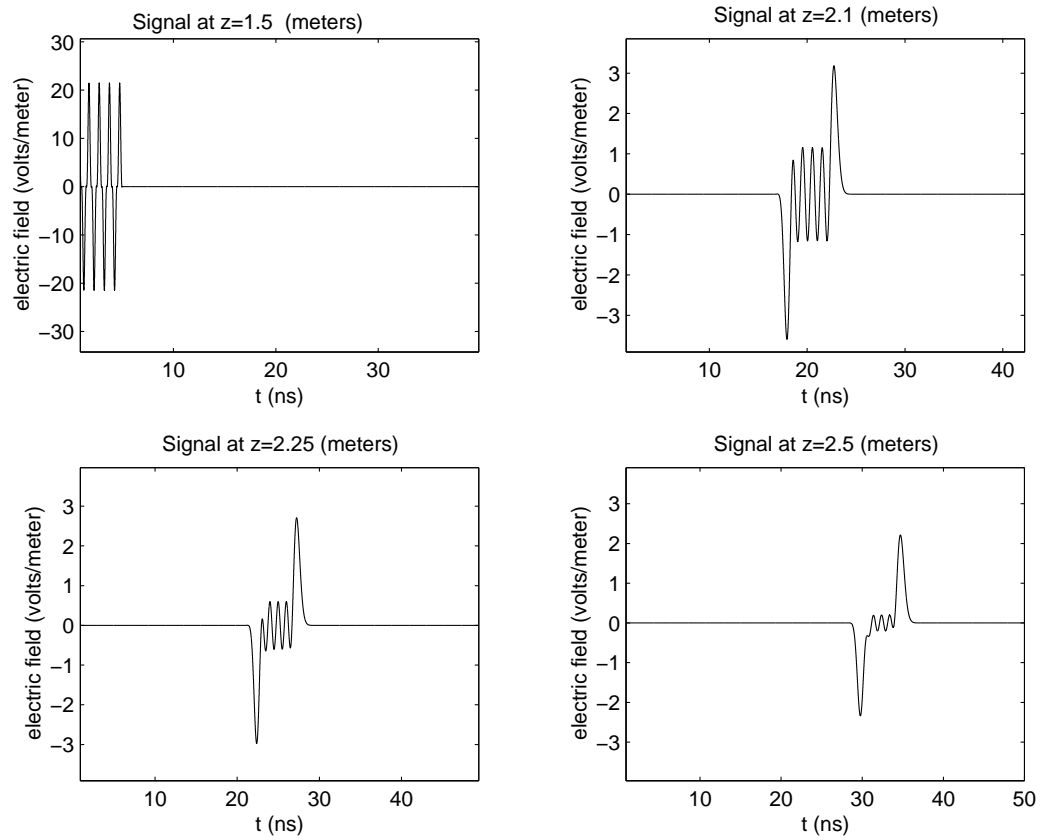

Figure 2: The electric field recorded at the antenna and at a distance of 0.6, 0.75 and $1 \mathrm{~m}$ from the antenna, $1 \mathrm{GHz}$ example. 

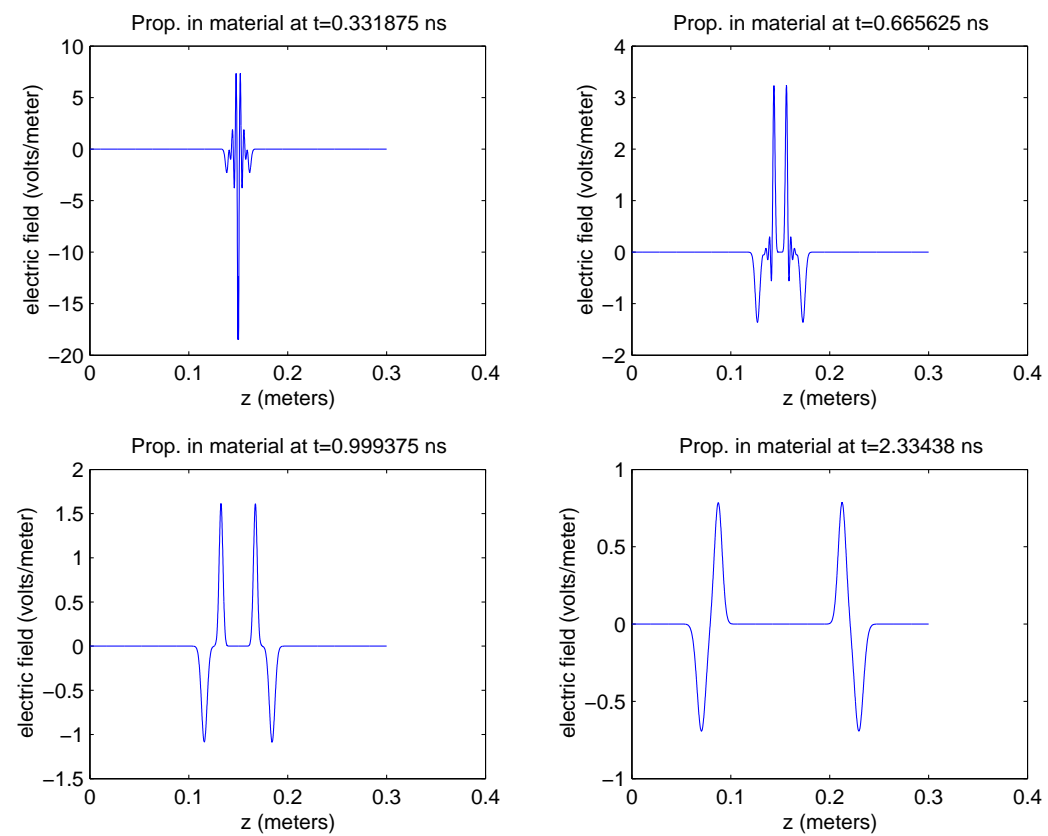

Figure 3: The electric field in the material at $\mathrm{t}=0.33,0.66,0.99$ and $2.33 \mathrm{~ns}, 10$ $\mathrm{GHz}$ example.
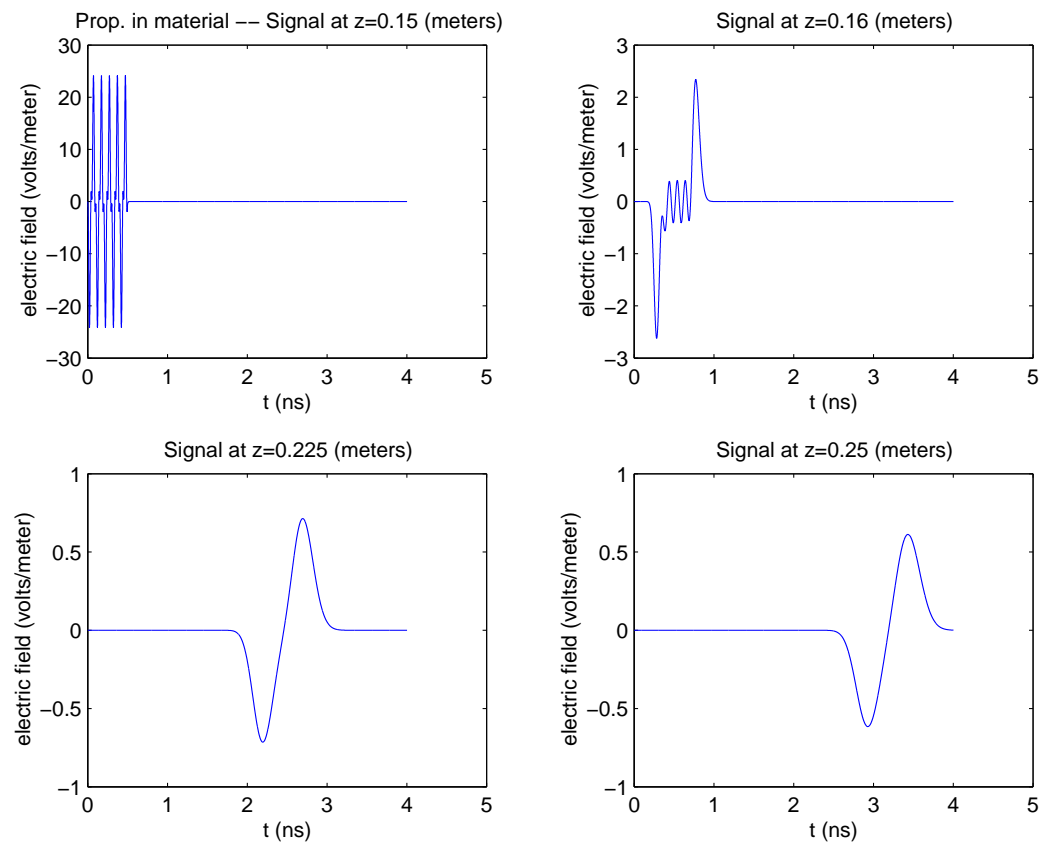

Figure 4: The electric field recorded at the antenna and at a distance of $1 \mathrm{~cm}$, $7.5 \mathrm{~cm}$ and $10 \mathrm{~cm}$ from the antenna, $10 \mathrm{GHz}$ example. 


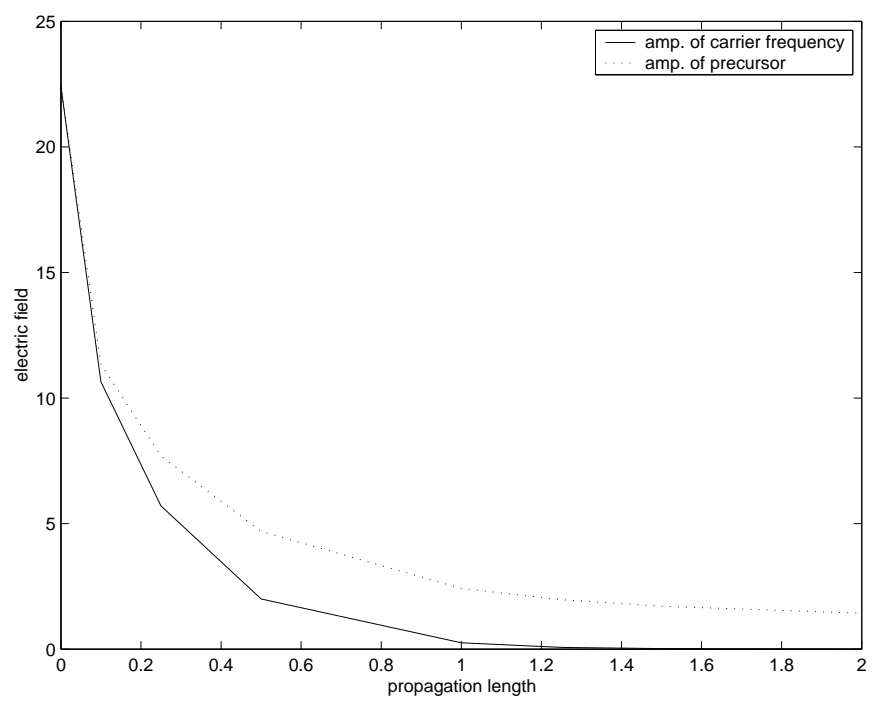

Figure 5: Attenuation of the peak of the transient (dashed line) and the carrier frequency (solid line).
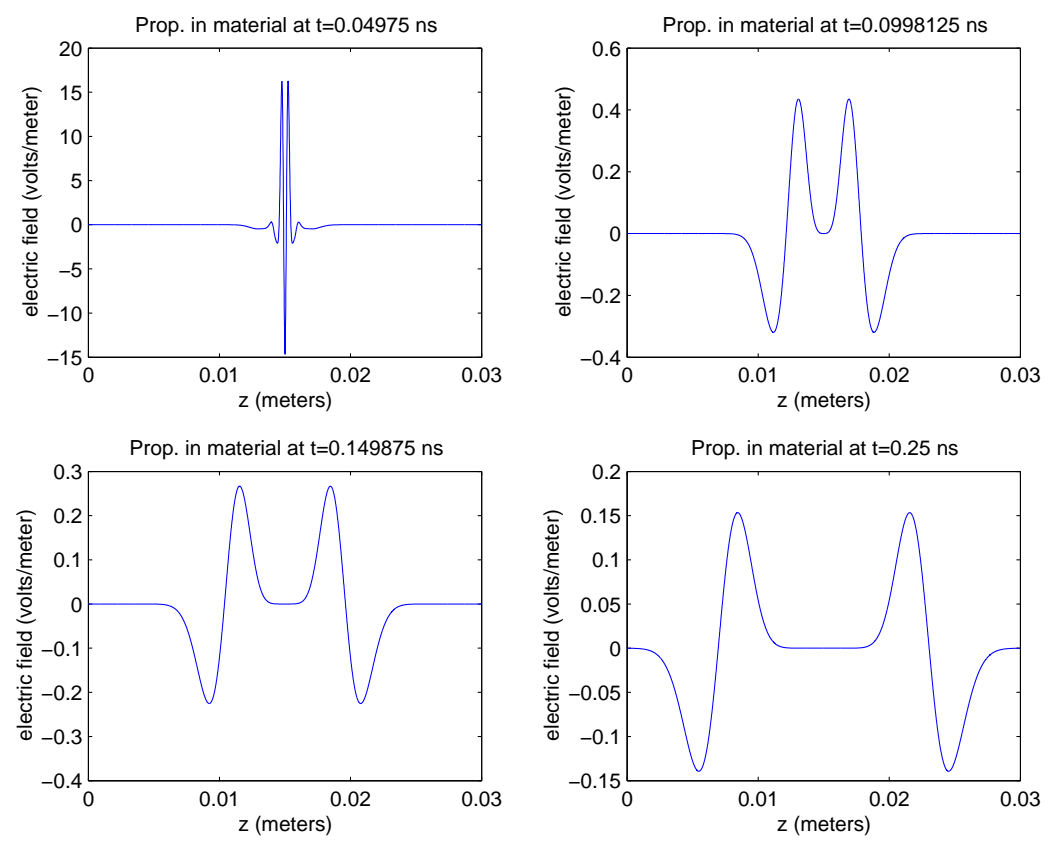

Figure 6: The electric field in the material at $\mathrm{t}=0.049,0.099,0.14$ and $0.25 \mathrm{~ns}, 100$ $\mathrm{GHz}$ example.

We can observe that the carrier frequency does not penetrate the material in these cases. It is only the transient that propagates inside the material. This result is expected based on theoretical considerations [8, p.313.] and experimental observations 

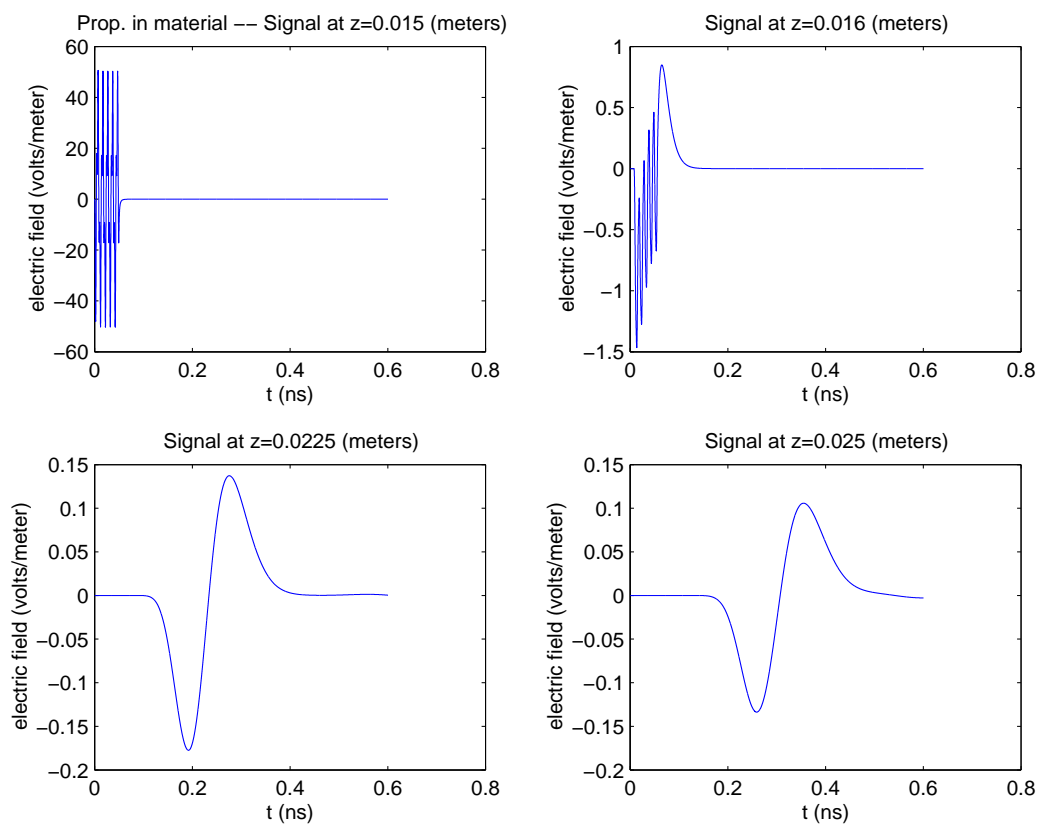

Figure 7: The electric field recorded at the antenna and at a distance of $1 \mathrm{~mm}, 7.5$ $\mathrm{mm}$ and $10 \mathrm{~mm}$ from the antenna, $100 \mathrm{GHz}$ example.
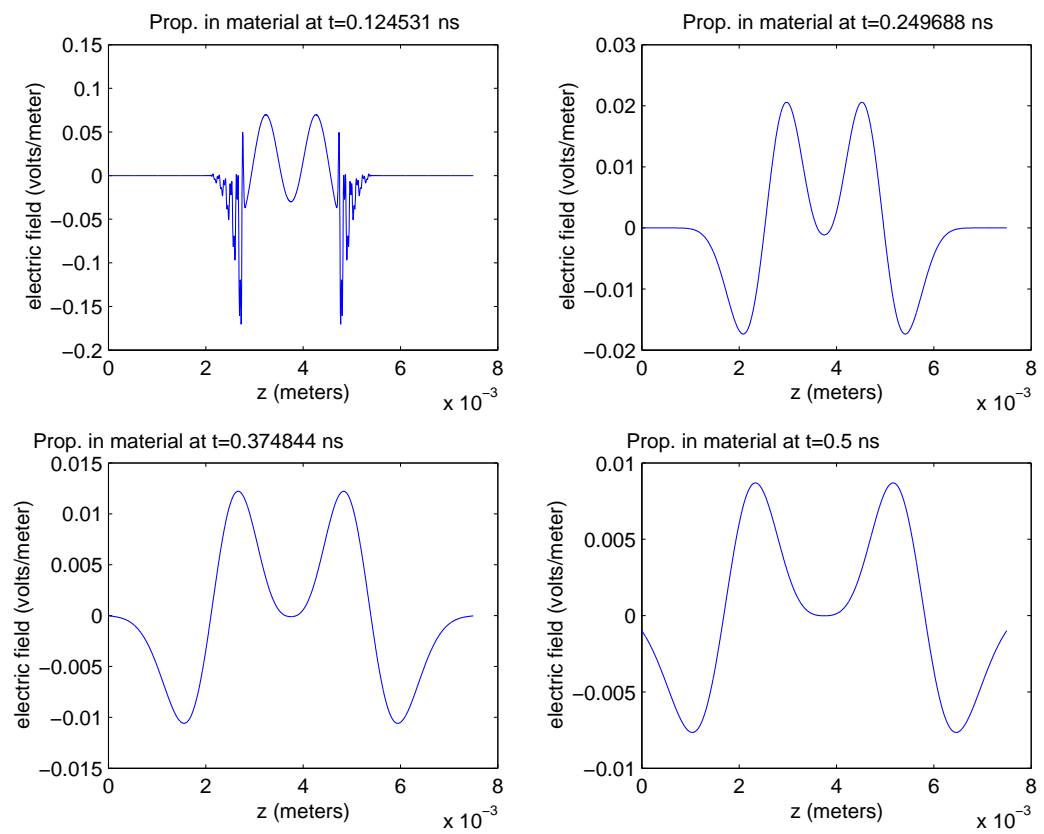

Figure 8: The electric field in the material at $\mathrm{t}=0.12,0.24,0.37$ and $0.5 \mathrm{~ns}, 1 \mathrm{THz}$ example. 

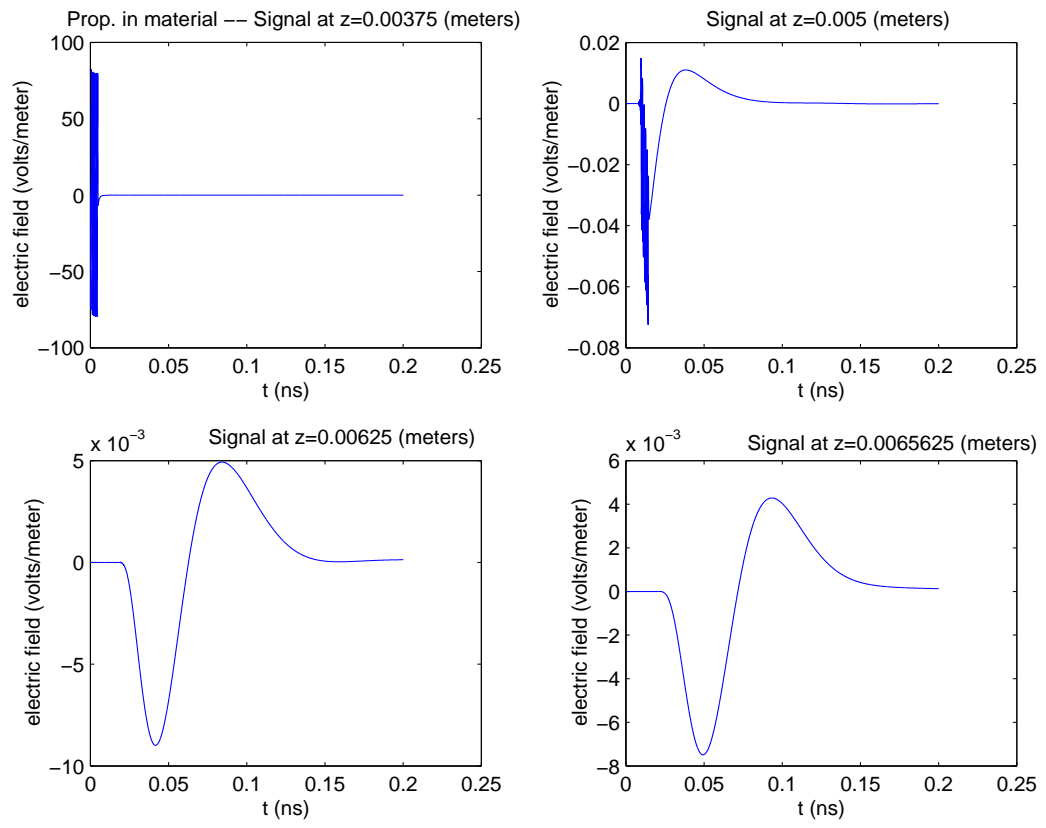

Figure 9: The electric field recorded at the antenna and at a distance of $1.25 \mathrm{~mm}$, $2.5 \mathrm{~mm}$ and $2.81 \mathrm{~mm}$ from the antenna, $1 \mathrm{THz}$ example.

$[11]$.

Thus we can see that our numerical simulations based on a variational formulation provide a detailed description of the propagation of the electromagnetic pulse, in particular, we are readily able to capture the temporal transients, such as the Brillouin precursors.

\section{Nonlinearly forced Debye polarization model}

In this section we introduce a polarization dynamics driven by a nonlinear function of the electric field. It is known that most materials exhibit nonlinear polarization characteristics especially when the amplitude of the input signal is large. In a first attempt to incorporate nonlinear effects in the Debye polarization model we considered

$$
\tau \dot{P}+P=\varepsilon_{0} \varepsilon_{d} E+f(E) .
$$

We showed that this constitutive relationship together with the weak formulation of the problem (2.8) is well-posed. In particular, we have the following result

Theorem 3.1 If the nonlinear function $f: \mathbb{R} \rightarrow \mathbb{R}$ is $C^{1}$, with $f(0)=0$, and $f^{\prime}(z)>$ 0 for all $z \in \mathbb{R}$, and $f$ is affine at infinity, i.e., there exist constants $R, L$ such that for every $|x|>R,|f(x)| \leq L|x|$, then there exists a weak solution to the system (2.8), (3.1) with initial conditions

$$
E(0, z)=\Phi,
$$




$$
\dot{E}(0, z)=\Psi
$$

for any $\Phi \in V, \Psi \in H, J_{s} \in H^{2}\left(0, T ; V^{*}\right)$. The weak solution is unique, and depends continuously on $\Phi, \Psi$ and $J_{s}$ under the additional assumption that $f$ is globally Lipschitz.

The details of the proofs can be found in [6].

In our numerical simulations we take $f(E)=\beta E^{3}$, which is an approximation to a saturation limited nonlinearity required by the above theorem. We implemented the same numerical method outlined in the previous section, except that in this case (2.14)-(2.15) contain nonlinear terms. We used a functional iteration in the nonlinear version of (2.14) to obtain $e^{n}$, which is then used to update the polarization term $p^{n}$. Comparison of the nonlinearly forced model (3.1) with the corresponding linear dynamics is depicted in Figures 11 and 12 for the $10 \mathrm{GHz}$ and $1 \mathrm{THz}$ case, respectively.

In these simulations we have a weak nonlinearity with $\beta=-5 \times 10^{-6}$ and the
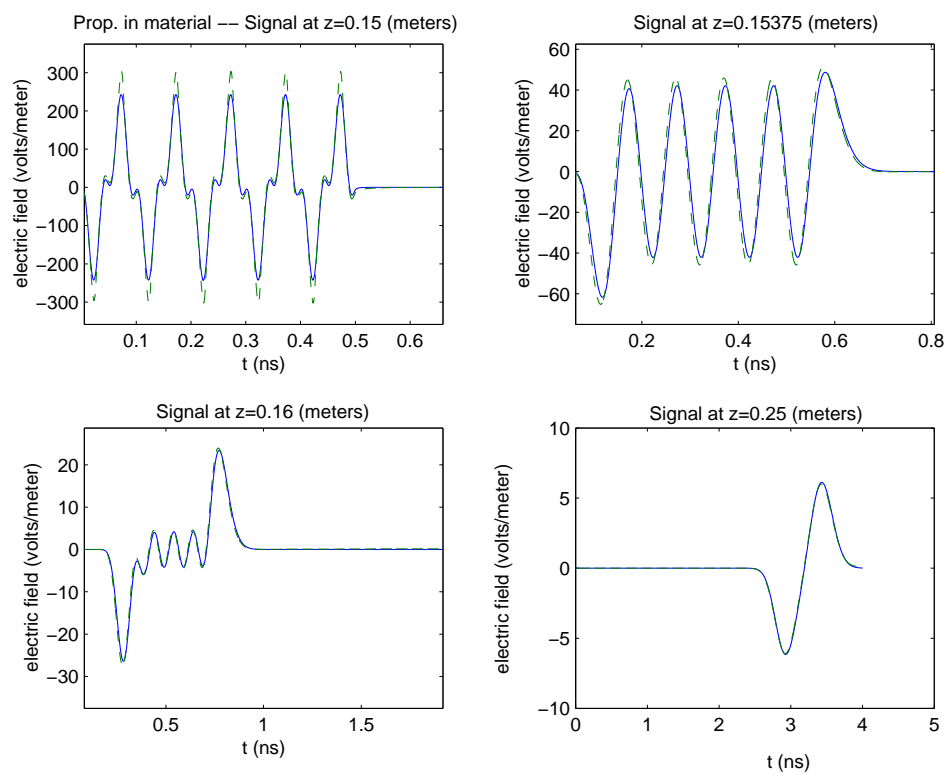

Figure 10: Comparison of the linear (solid line) and nonlinearly forced (dashed line) results, $10 \mathrm{GHz}$ example.

amplitude of the input signal is small, $A=10$. The linear and nonlinear results do not differ substantially in the $10 \mathrm{GHz}$ case (see Figure 11). In that case if $\beta$ is positive we observed that a nonlinearly forced polarization dynamics leads to a signal whose main part arrives slightly earlier and is slightly larger than the corresponding portion of the signal in the linear material. The reverse is true if the $E^{3}$ term has a negative coefficient in (3.1). The same observation holds true for the case of input signals with carrier frequency $1 \mathrm{GHz}$. However, in the $1 \mathrm{THz}$ example (see Figure 12), the situation is different. The main part of the signal arrives at approximately the same time in the linear and nonlinearly forced cases, and we can see a larger difference between the linear and nonlinearly forced results. 

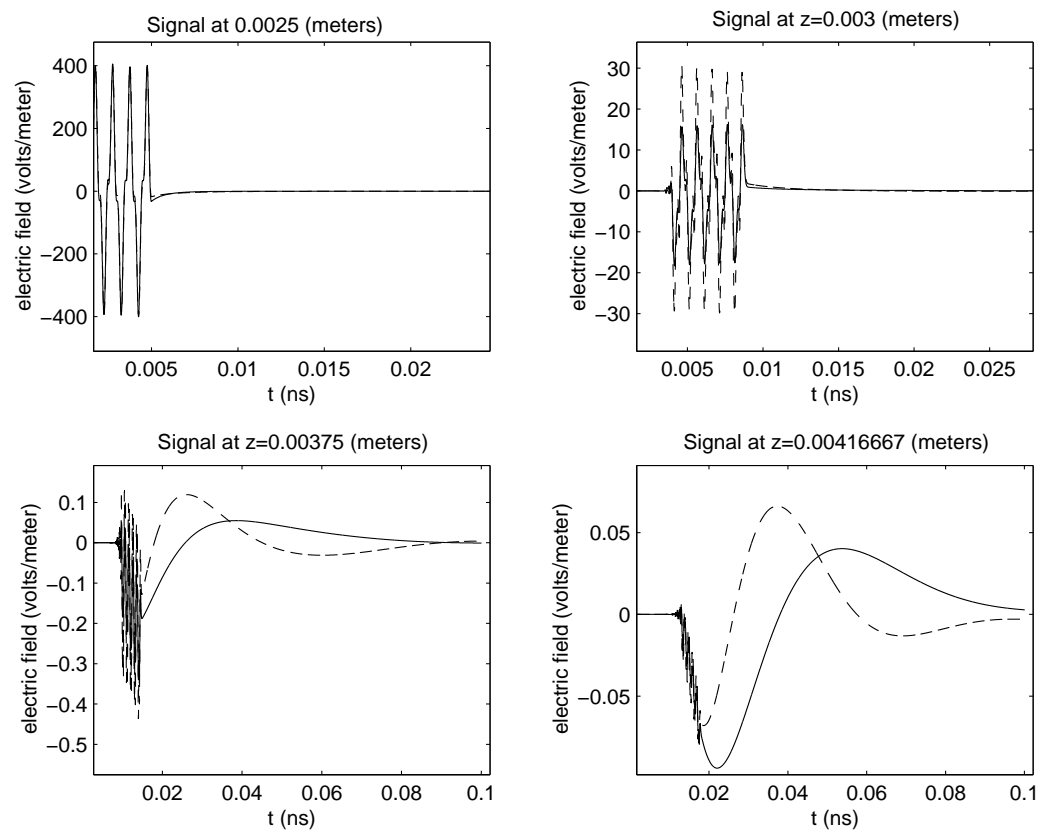

Figure 11: Comparison of the linear (solid line) and nonlinearly forced (dashed line) results, $1 \mathrm{THz}$ example.

\section{Nonlinear Debye Polarization model}

Our last set of numerical simulations are based on a nonlinear Debye medium with polarization dynamics given by:

$$
\tau \dot{P}+g(P)=\varepsilon_{0}\left(\varepsilon_{s}-\varepsilon_{\infty}\right) E .
$$

We choose $g(P)=P+s P^{3}$, where $s$ is a small parameter. The cubic nonlinear term is chosen since most biological tissues are not expected to have an axis of symmetry. However, it must be considered as an approximation to a saturation limited nonlinear mechanism that can be anticipated for orientational polarization. Large departures from the linear behavior can be observed even for weak nonlinearities if the amplitude of the input signal is sufficiently large. In our simulations $s=10^{-3}$ and the amplitude A of the input signal is in the range $10^{10}$ to $10^{12}$. Figures 13 and 14 show the comparison between the nonlinear Debye model (4.1) and the corresponding linear dynamics in the $10 \mathrm{GHz}$ and $1 \mathrm{THz}$ case, respectively.

We make several interesting observations. With $A=10^{10}$ in the $10 \mathrm{GHz}$ example (Figure 13), it is clear that the leading edge of the main part of the signal (i.e., the Brillouin precursor) arrives faster than in the corresponding linear simulation. Moreover, the electric field is considerably larger in the nonlinear Debye medium. However, an input signal with amplitude $A=10^{10}$ leads to no difference in the electric field in the linear and nonlinear simulations in the $1 \mathrm{THz}$ example (not shown) and one must increase the amplitude to see any difference in the results (see Figure 14, where $A=10^{11}$ ). The difference is similar to that we observed in the nonlinearly forced case 

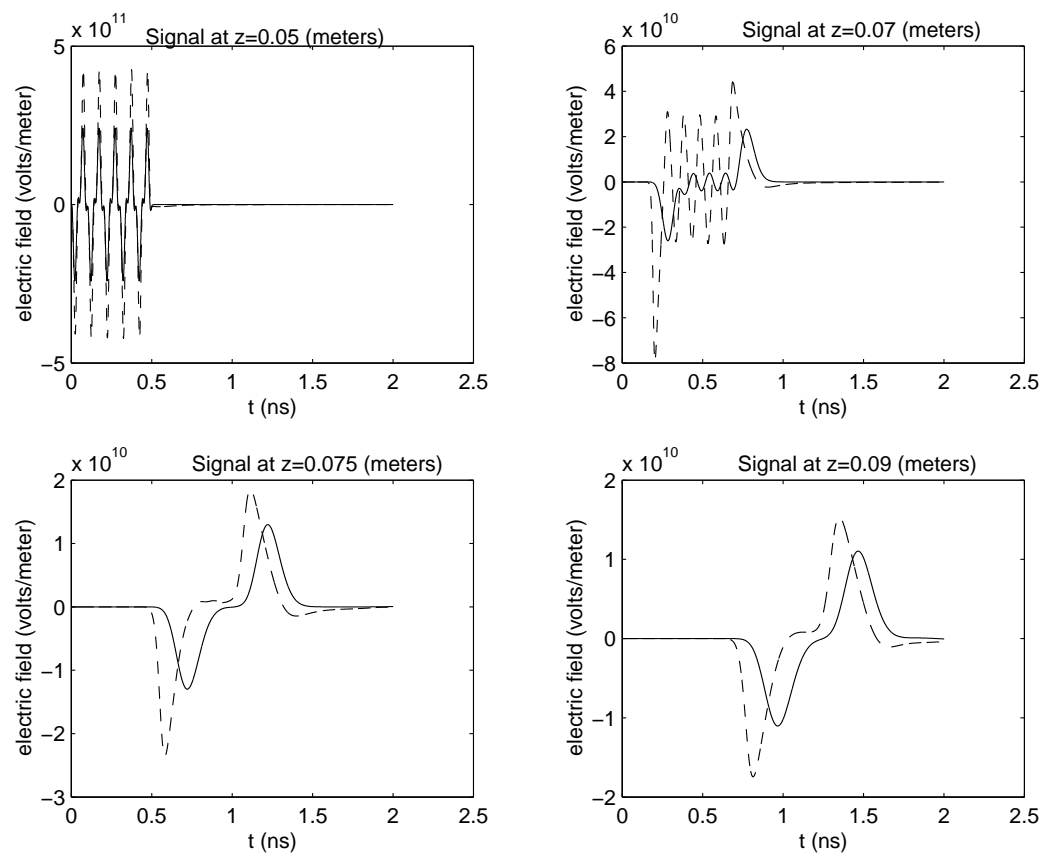

Figure 12: Comparison of the linear (solid line) and nonlinear (dashed line) results, $10 \mathrm{GHz}$ example.

in the terahertz regime. The main part of the signal appears at the same time, but in the nonlinear material the field is much larger, almost twice as large as in the linear case. We note that for $A=10^{12}$ the field produced in the nonlinear case is two orders of magnitude larger than that in the corresponding linear case.

\section{Conclusion}

In this paper we presented numerical results for the propagation of high frequency windowed pulses in dielectric materials. Although the problem was simplified by generating the signal inside the material we emphasize that this formulation can readily be extended to treat interfaces and reflected signals, and is therefore suitable to treat the inverse or interrogation problem. The understanding and correct description of temporal transients is important in its own right in assessing safety standards and making the interrogation problem practically feasible. As we noted in the Introduction, electromagnetic interrogation with high frequency windowed pulses have many potential applications. We remark that it is desirable to extend the physical problem to two and three dimensions, to create more realistic models for these applications. The two dimensional extension has been carried out for a linear Debye medium in [5] using perfectly matched layers. It was also implemented under slightly different assumptions and using a different numerical method in [4]. 

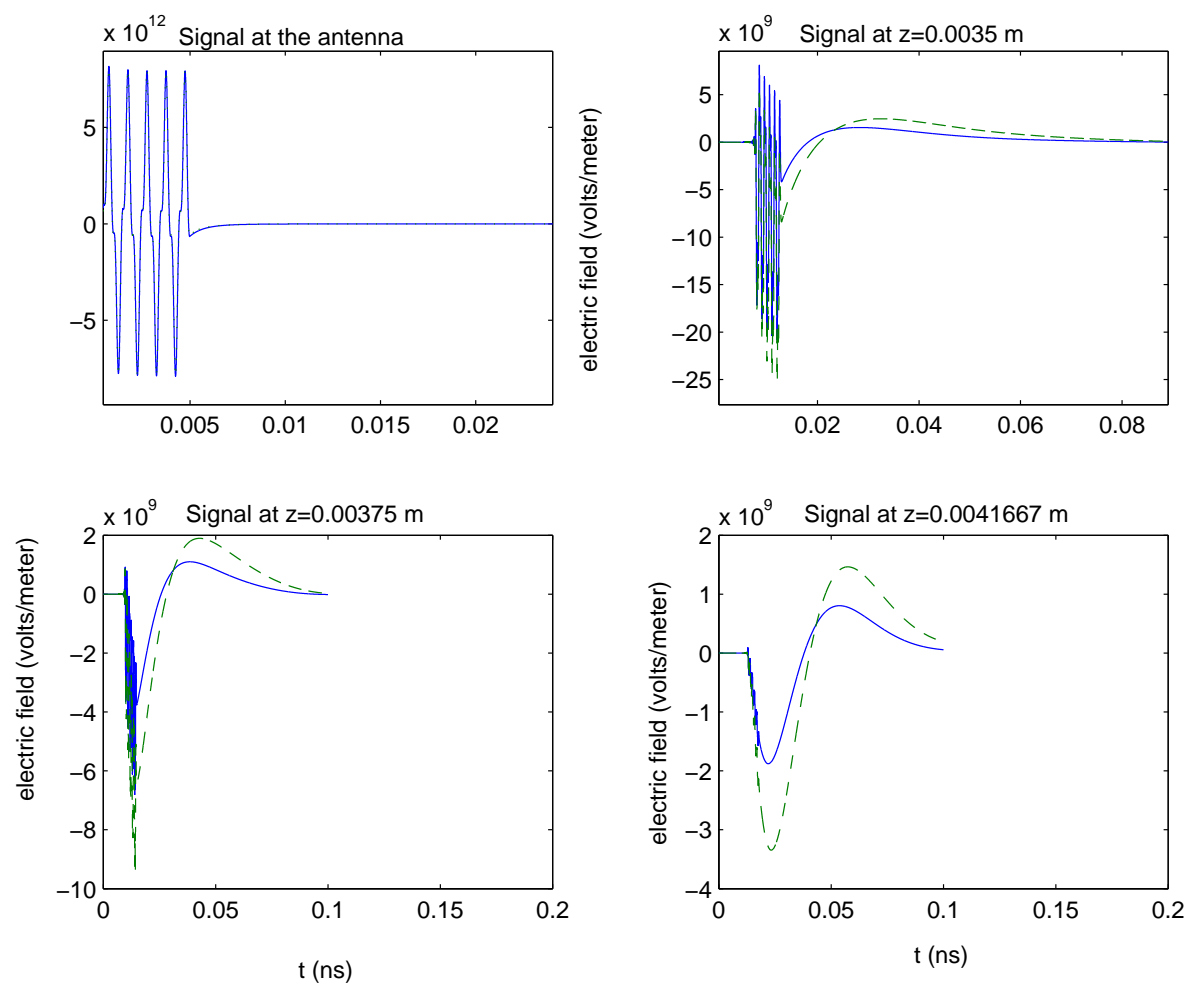

Figure 13: Comparison of the linear (solid line) and nonlinear (dashed line) results, $1 \mathrm{THz}$ example.

\section{Acknowledgments}

We are grateful to Dr. Richard Albanese for numerous stimulating conversations regarding the efforts reported on here.

This research was supported in part by the U.S. Air Force Office of Scientific Research under grants AFOSR F49620-01-1-0026 (H.T.B.) and AFOSR F49620-03-1-0185 (G.A.P.) and was facilitated through visits of the authors to the Statistical and Applied Mathematical Sciences Institute, (SAMSI), which is funded by NSF under grant DMS-0112069.

\section{References}

[1] R. Albanese, J. Penn and R. Medina, Short-rise-time microwave pulse propagation through dispersive biological media, Journal of the Optical Society of America A, 9, 1989, p.1441-1446.

[2] R. Albanese, J. Penn and R. Medina, Ultrashort pulse response in nonlinear dispersive media, Ultra-Wideband, Short-Pulse Electromagnetics, H. Bertoni et al. eds. , Plenum Press, 1993, p.259-264. 
[3] H.T. Banks, M.W. Buksas and T. Lin, Electromagnetic Material Interrogation Using Conductive Interfaces and Acoustic Wavefronts, SIAM Frontiers in Applied Mathematics, Philadelphia, 2000.

[4] H.T. Banks and J. Bardsley, Parameter identification for a dispersive dielectric in 2-D electromagnetics: forward and inverse methodology with statistical considerations, prerint, August 2003.

[5] H.T. Banks and B.L. Browning, Time domain electromagnetic scattering using finite elements and perfectly matched layers, CRSC-02-23, July, 2002; Computer Methods in Applied Mechanics and Engineering, submitted.

[6] H.T. Banks and G.A. Pinter, Maxwell-systems with nonlinear polarization, Nonlinear Analysis, Real World Applications, 4, 2003, p.483-501.

[7] P. Debye, Polar molecules, Chemical Catalog Co., New York, 1929.

[8] J.D. Jackson, Classical Electrodynamics, John Wiley and Sons, New York, 1975.

[9] D.L. Mills, Nonlinear Optics, Springer-Verlag, Berlin, 1991.

[10] A.C. Newell and J.V. Moloney, Nonlinear Optics, Addison-Wesley, Redwood City, 1991.

[11] P. Pleshko and I. Palocz, Experimental observation of Sommerfeld and Brillouin precursors in the microwave domain, Physical Review Letters, 22, 1969, p.1201-1204.

[12] Y.R. Shen Principles of Nonlinear Optics, Wiley, New York, 1984.

[13] C. Sulem and P-L. Sulem, The Nonlinear Schrödinger Equation, Self-Focusing and Wave Collapse, Springer-Verlag, New York, 1999. 